\title{
A Three-Dimensional Magnetohydrodynamic (MHD) Model of Active Region Evolution
}

\begin{abstract}
S. T. Wu ${ }^{1,2}$, A. H. Wang ${ }^{1}$, and D. A. Falconer ${ }^{3,4}$
${ }^{1}$ Center for Space Plasma and Aeronomic Research, ${ }^{2}$ Department of Mechanical and Aerospace Engineering, ${ }^{3}$ Department of Physics, University of Alabama in Huntsville, Huntsville, AL 35899 USA email: wus@cspar.uah.edu,wanga@cspar.uah.edu, David.Falconer@msfc.nasa.gov

${ }^{4}$ XD12, NASA/Marshall Space Flight Center, Huntsville, AL 35812 USA

Abstract. A three-dimensional, time-dependent, magnetohydrodynamic (MHD) model is constructed for the study of active region (AR) evolution. The new physics included in this model is differential rotation, meridional flow, effective diffusion and cyclonic turbulence effects, which means, that the photospheric shear is automatically generated instead of prescribed as is usually done for modeling. To benchmark this newly developed model, we have used observed active region NOAA/AR-8100 (October 29 - November 3, 1997) to verify the model by computation of the total magnetic flux and magnetic field maps of that active region. Then, we apply this model to compute the non-potentiality magnetic field parameters for possible coronal mass ejection production. These parameters are: (i) magnetic flux content $(\Phi)$, (ii) the length of strong shear, strong-field main neutral line, $\left(L_{s s}\right)$, (iii) the net electric current $\left(I_{N}\right)$ and (iv) the flux normalized measure of the field twist $\left(\alpha=\mu \frac{I_{N}}{\Phi}\right)$. These parameters are compared with the measured values which showed remarkable agreement.
\end{abstract}

Keywords. Sun:Active Region, Sun:MHD, Sun:Coronal Mass Ejections (CMEs), Sun:Flares, Sun:Magnetic Fields

\section{Introduction}

Understanding the sources of solar eruptive phenomena requires knowledge of the evolution of the active region. By looking at the full disk of the photospheric magnetogram, it is immediately recognized that the evolution of sunspots and sunspot groups are the sources of the most powerful solar eruptions (Wang et al. 2002, Wang et al. 2004). In an early study, Leighton (1964) modeled the sunspots and solar cycle in relation to the expansion and migration of unipolar (UM) and bipolar (BM) magnetic regions. Since then, a number of investigators (DeVore, et al. 1984; McIntosh \& Wilson, 1985; Sheeley, et al. 1985; Sheeley \& Devore 1986; Wilson \& McIntosh, 1991; Wang \& Sheeley, 1991; McKay 2003) have extensively investigated the magnetic flux transport in relation to the solar cycle by means of a modified Leighton model with additional physics. Wang and Sheeley (1991) have presented a numerical simulation including differential rotation, supergranular diffusion, and a poleward surface flow (i.e. meridional flow) of the redistribution of magnetic flux erupting in the form of bipolar magnetic regions (BMRs). They reproduced many of the observed features of the Sun's large scale field not encompassed by the Leighton (1964) model. Wilson and McIntosh (1991) compared observed evolution of large-scale magnetic fields with simulated evolution based on the kinematic model of Devore \& Sheeley (1987). They concluded that there must be significant contributions to the evolving patterns by non-random flux eruptions within the network structure, independent of active regions. McKay (2003) presented a magnetic flux transport simulation of the Sun's surface distribution of magnetic fields during Maunder minimum. All 
these works are focused on the large-scale field and long-time-scale (i.e. solar cycle) evolution. In the case of small-scale field and short-time-scale (i.e. hours and days), the basic flux transport model was also applied with additional physical features (Schrijver, 2001; Schrijver \& Title, 2001). However, all these investigations have not invoked full magnetohydrodynamic (MHD) theory; that means the nonlinear dynamic interactions among the plasma flow field and magnetic field are ignored. In order to include this nonlinear dynamic interaction, $\mathrm{Wu}$, et al. (1993) have constructed a quasi-three-dimensional, time-dependent incompressible MHD model with differential rotation, meridional flow and effective diffusion as well as cyclonic turbulence to study evolution of BMRs. In their limited quasi-three-dimensional theoretical study, they have demonstrated that the observed complexity pattern could arise on the Sun's surface due to the dynamic interaction between the flow fields and magnetic field (i.e. MHD effect) and growth and decay of a BMR.

In this paper we will present a full three-dimensional, time-dependent, compressible MHD model with differential rotation, meridional flow, effective diffusion due to random motion of the granules or the super-granules and cyclonic turbulence effect to study the active region evolution to deduce the non-potential magnetic field parameters for possible initiation of solar eruptive events using observed magnetic field data as the initial conditions. The mathematical model, initial and boundary conditions are presented in Section 2, numerical results and concluding remarks are given in Section 3 and 4, respectively.

\section{Mathematical Model, Initial and Boundary Conditions}

\subsection{Mathematical Model}

On the basis of magnetohydrodynamic (MHD) theory, the mathematical model appropriate for the physical scenario we described in the previous section can be expressed by a set of compressible MHD equations consisting of conservation of mass, momentum, energy and the induction equation resulting from Maxwell's equations. These equations account for non-linear dynamic interactions of plasma flow and magnetic field. These governing equations are:

$$
\begin{gathered}
\frac{\partial \rho}{\partial t}+\nabla \cdot(\rho \vec{u})=0 \\
\rho\left[\frac{\partial \vec{u}}{\partial t}+\vec{u} \cdot \nabla \vec{u}\right]=-\nabla p+\frac{1}{4 \pi}(\nabla \times \vec{B}) \times \vec{B}+\vec{F}_{g}-2 \pi \vec{\omega}_{o} \times \vec{u}-\rho \vec{\omega}_{o} \times\left(\vec{\omega}_{o} \times \vec{r}\right)+\Psi \\
\text { where, } \Psi=-\frac{2}{3} \nabla(\mu \nabla \cdot \vec{u})+\mu\left[\nabla^{2} \vec{u}+\nabla(\nabla \cdot \vec{u})\right]+2[(\nabla \mu) \cdot \nabla] \vec{u}+[(\nabla \mu) \times(\nabla \times \vec{u})] \\
\frac{\partial p}{\partial t}+\vec{u} \cdot \nabla p+\gamma p \nabla \cdot \vec{u}=(\gamma-1) \nabla \cdot \vec{Q}+(\gamma-1)\left[\eta J^{2}+\frac{\mu}{2}(\nabla \cdot \vec{u})^{2}\right] \\
\frac{\partial \vec{B}}{\partial t}=\nabla \times(\vec{u} \times \vec{B})+\lambda(\nabla \times \vec{B})+\eta \nabla^{2} \vec{B}+\vec{S}
\end{gathered}
$$

where $\rho$ is the plasma mass density, $\vec{u}$ the plasma flow velocity vector, $p$ the plasma thermal pressure, $\vec{B}$ the magnetic induction vector, $\vec{J}$ the electric current and $\vec{S}$ the energy source term, respectively. The other quantities are defined as follows: $\vec{\omega}_{o}$ is the angular velocity of solar differential rotation referring to the center of the solar coordinate system, 
that is given by Snodgrass (1983) and the meridional flow profile used here is given by Hathaway, (1996). $\vec{F} g$ is the gravitational force, $\nabla \cdot \vec{Q}$ represents the heat conduction, $\gamma, \mu, \lambda$, and $\eta$ are the specific heats ratio (1.05), viscosity, coefficients of the cyclonic turbulence and effective diffusion. Finally, the $\Psi$ represents the viscous dissipation.

This set of MHD equations differs from first principle MHD theory because of the inclusion of additional physics. For example, the additional terms in $\mathrm{Eq}(2.2)$ represent the inertial centrifugal force (i.e. $\left.2 \rho \vec{\omega}_{o} \times \vec{u}\right)$ and the coriolis force $\left(2 \rho \vec{\omega}_{o} \times \vec{\omega}_{o} \times \vec{r}\right)$ due to the Sun's differential rotation. The terms $\eta \nabla^{2} \vec{B}$ and $\lambda(\nabla \times \vec{B})$ in Eq. (2.4) represent the effective diffusion due to random motion of granules or super-granules and cyclonic turbulence effect, respectively.

\subsection{Initial and Boundary Conditions}

To simulate the active region evolution, we have cast the set of governing equations in a rectangular coordinate system. The computational domain includes six planes (i.e. four side planes, top and bottom). The boundary conditions used for the four sides are linear extrapolation, top boundary is non-reflective boundary and the bottom boundary is derived from the method of characteristics (Wu and Wang, 1987; Wang 1992) which is given in the Appendix. This set of boundary conditions is the time-dependent boundary conditions. In such a way, we are able to model the emerging and submerging magnetic flux in a self-consistent manner.

To implement this evolutionary simulation of the active region, we apply the following steps:

\subsubsection{Initializing the Simulation of the Active Region}

(a) Use the magnetic field data from photospheric magnetogram together with potential field model to construct a three-dimensional field configuration.

(b) Since there is no density measurement on the photosphere, we simply assume that the density distribution at the photospheric level is directly proportional to the absolute value of the magnitude of the transverse field and decreases exponentially with the scale height, thus $\rho(x, y, z, 0)=\sqrt{\frac{B_{x}^{2}+B_{y}^{2}}{B_{o}^{2}}} e^{-\frac{z}{H_{g}}}$ where $\rho_{o}$ and $\mathrm{B}_{o}$ are the constant reference values with $H_{g}$ as the scale height, and

(c) Input the results of (a) and (b) into the MHD model described in Section 2 to allow its relaxing to a quasi-equilibrium state. This will be our initial state for the study of the evolution.

\subsubsection{Evolutionary Simulation of the Active Region}

To evolve the corona, we apply differential rotation and meridian flow to the lower boundary (photospheric magnetic field) in 5-second time steps. After each time step, we allow the corona to respond to the changes in the lower boundary condition. Once, every $\sim 96$ minutes, when a new MDI magnetogram is available, we add or subtract magnetic flux at the lower boundary according to the expression given by Eq. (A6) to mimic the emergence and submergence of the magnetic flux where the first term represents the general increasing and decreasing of the magnetic flux in the whole region and the second term is represented by a "delta" function which takes account the pop-up flux at a specific location. The coefficient " $a$ " in Eq. (A6) is chosen according to the cadence of the observation and the computation time step. In this calculation $a$ is $10^{-4}$.

With these two procedures, we obtain the time and spatial evolution of the active region represented by this initial state. 

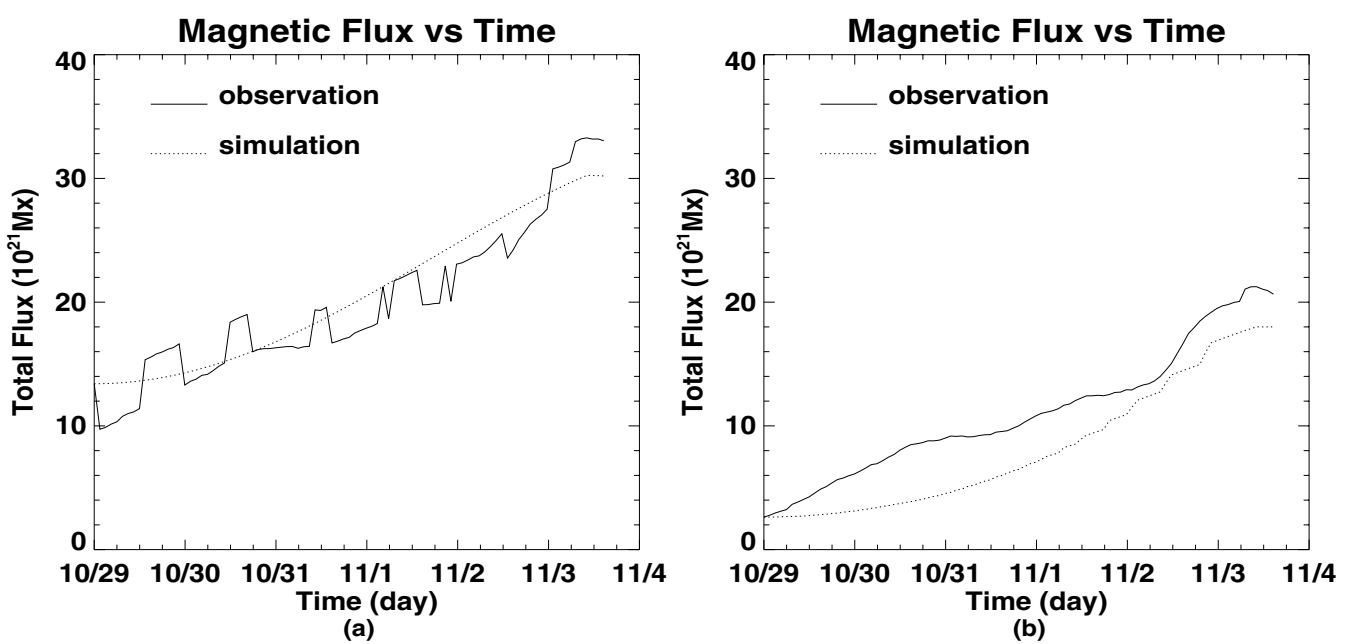

Figure 1. The simulated and measured (SOHO/MDI) magnetic flux content of AR8100 for (a) all fields and (b) strong fields (i.e. $|\vec{B}| \geqslant 100 \mathrm{G}$ ) during the period October 29 - November 3, 1997.

\section{Numerical Results}

To carry out this simulation study, we have chosen the SOHO/MDI magnetic field measurements of NOAA/AR 8100 from October 29, 11:15 UT to Nov 3, 15:59UT, 1997 for this study. The SOHO/MDI field measurements of the active region have a resolution of $\sim 2$ arc sec with $198 \times 198$ pixels with a candence of $\sim 96$ min. In order to assure the computational grids are compatible with the measurements, the computational domain is set as a rectangular region with $99 \times 99 \times 99$ grid points in Carrington longitude $(x)$, latitudinal direction $(y)$ and height $(z)$, respectively. To match the data with the grids, we have taken four point average of the pixels inside the domain. On the boundary we have taken a two point average from the measurements. At the four corners, the measurements are used. Before we can carry out the simulation study, we need to know two important coefficients; effective diffusivity $(\eta)$ and cyclonic turbulence $(\lambda)$. There are no precise theory and observations and laboratory experiments to determine these coefficients. However, there are some previous works which have discussed the choice of these two coefficients. For example, $\eta=160-300 \mathrm{~km}^{2} \mathrm{~s}^{-1}$ given by Parker, (1979); Leighton's value of $\eta$ is $800-1600 \mathrm{~km}^{2} \mathrm{~s}^{-1}$ (1964); DeVore, et al. (1985) selected $\eta=$ $300 \mathrm{~km}^{2} \mathrm{~s}^{-1}$ for their study. Wang (1988) derived a value of $\eta$ being $100-150 \mathrm{~km}^{2} \mathrm{~s}^{-1}$ on the basis of observation of sunspot's decay. We noticed that there is a wide range of values for the effective diffusivity. The value of cyclonic turbulence is chosen according to the scale law $(\lambda \sim \eta / L)$, given by Parker, (1979) where $L$ is the characteristic length of the sunspot, it is chosen to be $6,000 \mathrm{~km}$ for this study and $\eta$ is $200 \mathrm{~km}^{2} \mathrm{~s}^{-1}$.

\subsection{Model Verification}

Since there is no analytical solution to test this three-dimensional time-dependent MHD model of active region evolution, the only test which could be accomplished is to use observations. To carry out this test, we compare the simulated total flux content and the contours of the line-of-sight component of the magnetic field of the active region with the observation. Figure 1 shows the simulated and measured total flux content of NOAA/AR8100 during the period of October 29 - November 3, 1997 for strong fields (Fig1(b)) and all fields (Fig 1(a)), respectively. Simulated total flux content is obtained 
B_r Contour 1997/10/31 11:15UT

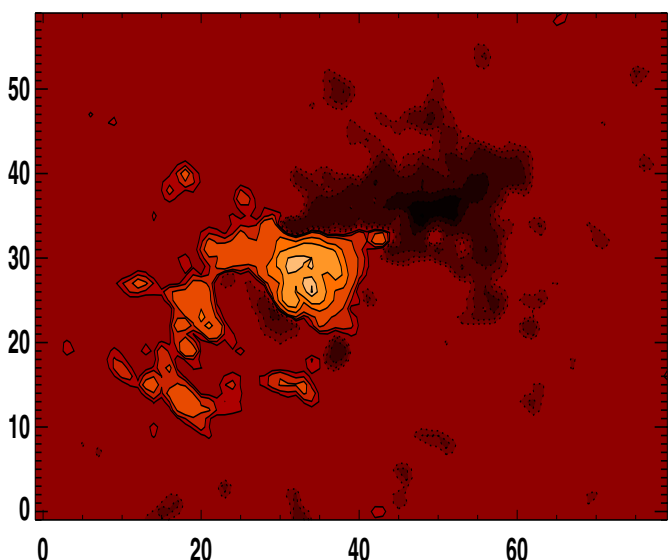

simulated

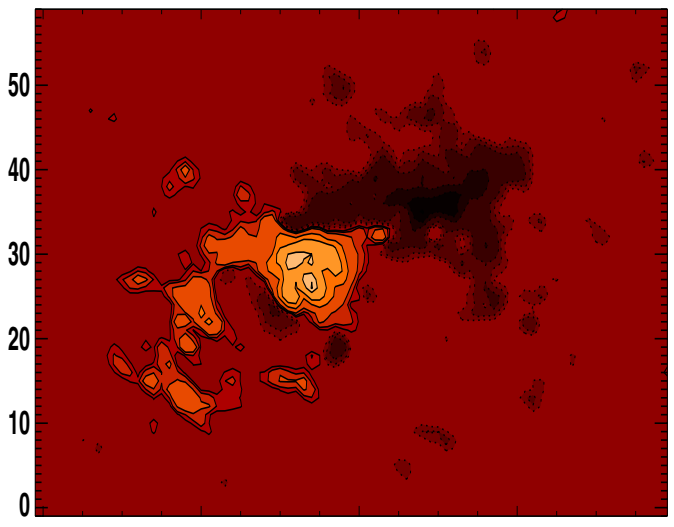

020

40

60
0

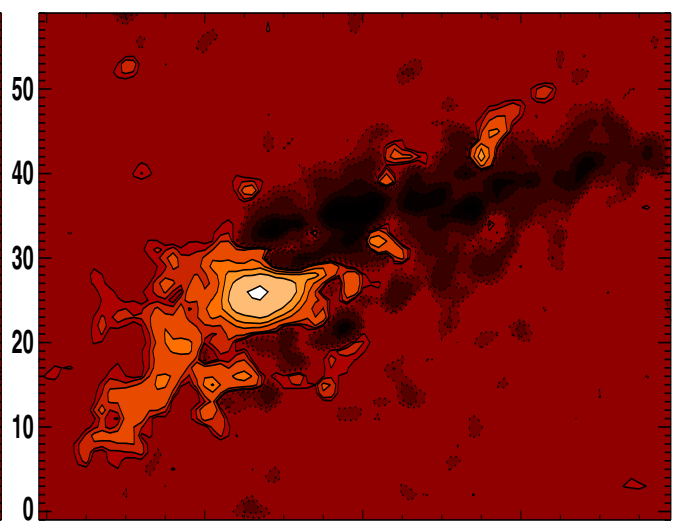

B_r Contour 1997/11/03 15:59UT

20

40

60

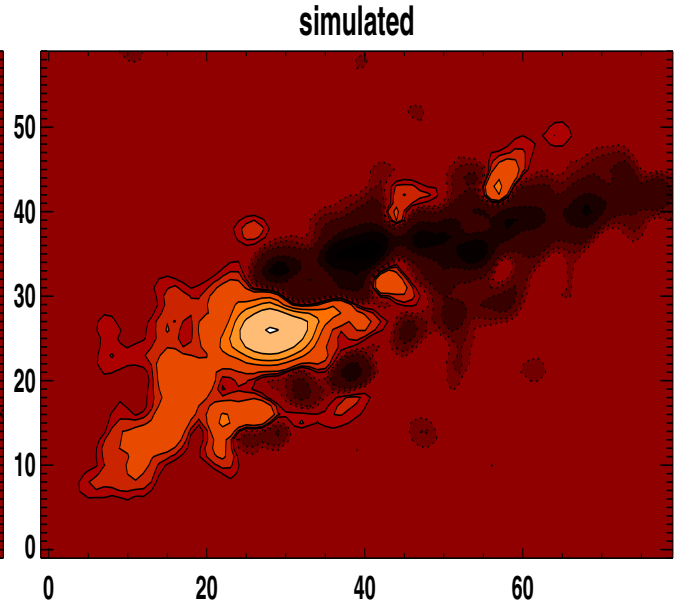

Figure 2. Comparison between the SOHO/MDI measured (upper panel) and simulated (lower panel) contours of the line-of-sight magnetic field maps at 1997 Oct 31, 11:15 UT and 1997 Nov 3, 15:50 UT, respectively for AR8100.

by input of the SOHO/MDI measured line-of-sight magnetic field together with potential field model into the three-dimensional, MHD AR evolution model described in Section 2. Then, the total flux content of the region is computed (i.e. $\Phi=\int_{A} \vec{B} \cdot d \vec{A}$; where $\vec{A}$ is the area of AR). The measured total flux content is obtained by computing $\Phi=\int_{A} \vec{B} \cdot d \vec{A}$ from the measured magnetic field at various time. Let us examine these results shown in Figure 1; it is clearly shown that the model simulated and measured total flux content $(\Phi)$ agree well. We also notice that the weak field does contribute significantly to the amount of total flux content.

Figure 2 shows the simulated and measured (SOHO/MDI) contours of the line-ofsight component of magnetic field at 1991 Oct 31, 11:15 UT and 1997 Nov 3, 15:59UT, respectively. Again the model results have mimicked the observed features well.

\subsection{Simulation of Non-potential Magnetic Field Parameters}

Using the definitions given by Falconer et al. (2002), the non-potential magnetic field parameters are computed based on the model outputs. These non-potential magnetic field 
Table 1. Non-Potential Magnetic Field Parameters of AR8100 during the Period of October 31 - November 3, 1997

\begin{tabular}{|c|c|c|c|c|c|c|c|c|c|}
\hline \multicolumn{2}{|c|}{$\begin{array}{c}\text { Time } \\
\text { (Date)|(UT) }\end{array}$} & \multicolumn{2}{|c|}{$\begin{array}{l}\mathrm{L}_{s s}\left(10^{3} \mathrm{~km}\right) \\
(\mathrm{Obs}) \quad(\mathrm{Sim})\end{array}$} & \multicolumn{2}{|c|}{$\begin{array}{c}\mathrm{I}_{N}\left(10^{11} \mathrm{~A}\right) \\
(\mathrm{Obs}) \mid(\mathrm{Sim})\end{array}$} & \multicolumn{2}{|c|}{$\begin{array}{l}\alpha\left(10^{-8} / \mathrm{m}\right) \\
(\mathrm{Obs}) \\
(\mathrm{Sim})\end{array}$} & \multicolumn{2}{|c|}{$\begin{array}{c}\Phi\left(10^{21} \mathrm{Mx}\right) \\
(\mathrm{Obs}) \mid(\mathrm{Sim})\end{array}$} \\
\hline $10 / 31$ & $11: 15$ & - & 0 & - & 0 & - & 0 & - & 5.8 \\
\hline $10 / 31$ & :51 & - & 12.6 & - & 0.4 & - & 0.050 & - & 5.9 \\
\hline $10 / 31$ & $14: 27$ & - & 20.0 & - & 0.7 & - & 0.080 & - & 6.0 \\
\hline $10 / 31$ & $15: 07$ & $28 \pm 8^{*}$ & 22.5 & $1 \pm 1^{*}$ & 0.8 & $0.13 \pm 0.1^{*}$ & 0.092 & $6 \pm 1^{*}$ & 6.2 \\
\hline $10 / 31$ & 16:03 & - & 24.0 & - & 0.9 & - & 0.105 & - & 6.3 \\
\hline $10 / 31$ & $17: 39$ & - & 27.0 & - & 1.1 & - & 0.120 & - & 6.4 \\
\hline $10 / 31$ & $20: 48$ & - & 29.4 & - & 1.3 & - & 0.140 & - & 6.8 \\
\hline - & - & - & - & - & - & - & - & - & - \\
\hline $11 / 01$ & 01:39 & - & 33.1 & - & 1.6 & - & 0.148 & - & 7.5 \\
\hline $11 / 01$ & $06: 27$ & - & 36.2 & - & 1.8 & - & 0.152 & - & 8.0 \\
\hline $11 / 01$ & $11: 25$ & - & 37.5 & - & 1.9 & - & 0.155 & - & 8.8 \\
\hline $11 / 01$ & $16: 03$ & - & 39.4 & - & 2.2 & - & 0.158 & - & 9.5 \\
\hline $11 / 01$ & $20: 48$ & - & 42.5 & - & 2.4 & - & 0.159 & - & 10.6 \\
\hline & & - & - & - & - & 一 & - & - & - \\
\hline $11 / 02$ & 01:39 & - & 41.5 & - & 2.5 & - & 0.161 & - & 11.7 \\
\hline $11 / 02$ & $06: 27$ & - & 48.0 & - & 2.7 & - & 0.165 & - & 12.6 \\
\hline $11 / 02$ & $11: 12$ & - & 51.0 & - & 3.0 & 一 & 0.172 & - & 14.0 \\
\hline $11 / 02$ & 16:00 & - & 54.1 & - & 3.2 & - & 0.181 & - & 14.7 \\
\hline $11 / 02$ & $20: 48$ & - & 57.2 & - & 3.6 & - & 0.192 & - & 15.8 \\
\hline - & & - & - & - & - & 一 & - & 一 & - \\
\hline $11 / 03$ & 01:39 & - & 61.6 & - & 4.1 & - & 0.218 & - & 16.8 \\
\hline $11 / 03$ & $06: 27$ & - & 67.5 & - & 4.8 & - & 0.260 & - & 17.7 \\
\hline $11 / 03$ & 09:37 & - & 71.4 & - & 5.4 & - & 0.295 & - & 18.0 \\
\hline $11 / 03$ & $12: 51$ & - & 77.0 & - & 6.5 & - & 0.354 & - & 18.1 \\
\hline $11 / 03$ & $14: 24$ & - & 81.6 & - & 7.5 & - & 0.392 & - & 18.0 \\
\hline $11 / 03$ & $14: 58$ & $85 \pm 17^{*}$ & 83.3 & $7 \pm 2^{*}$ & 7.8 & $0.44 \pm 0.1^{*}$ & 0.413 & $14 \pm 3^{*}$ & 17.9 \\
\hline $11 / 03$ & $15: 59$ & - & 86.4 & - & 8.8 & - & 0.460 & - & 17.7 \\
\hline
\end{tabular}

*These two data points are obtained from NASA/MSFC vector magnetogram (Falconer, et al 2002); Obs $=$ Observed and Sim $=$ simulated

parameters are; (i) total magnetic flux context, $(\Phi)$; (ii) the length of strong magnetic shear $\left(\geqslant 45^{\circ}\right)$ and strong transverse field $\left(\geqslant 150\right.$ gauss) of the main neutral line, $\left(\mathrm{L}_{s s}\right)$; (iii) the net electric current $\left(\mathrm{I}_{N}\right)$; and (iv) the flux normalized measure of the field twist $\left(\alpha=\mu \frac{I_{N}}{\Phi}\right)$. Table 1 shows these parameters as a function of time and these values are compared with the values given by Falconer et al. (2002) at two specific times. Their results are obtained by using the MSFC vector magnetograph data. There is no time series of observations with MSFC's vector magnetograms, thus, we can only make comparison with these two specific times. This is the reason why we have chosen SOHO/MDI data for this study because the SOHO/MDI has made continuous measurements. Examination of the tabulated values of non-potential parameters further shows that at the initial state (October 31, 11:15 UT), three of four non-potential parameters simulated are null because the initial state is approximated by potential model. As time progresses, all non-potential parameters increase due to the Sun's rotation and meridional flow as well as the flux emergence and submergence according to the effects of the lower boundary (i.e. photosphere shown in the Appendix). As such, the MHD effects occur due to the nonlinear dynamical interactions between the magnetic fields and plasma flow, in which, the magnetic shear is generated where the magnetic field is stressed, it leads to field expansion. Subsequently the mass is lifted up by the movement of magnetic field shown in Figure 3 and then partially opens up, which looks similar to the "break-out" model 

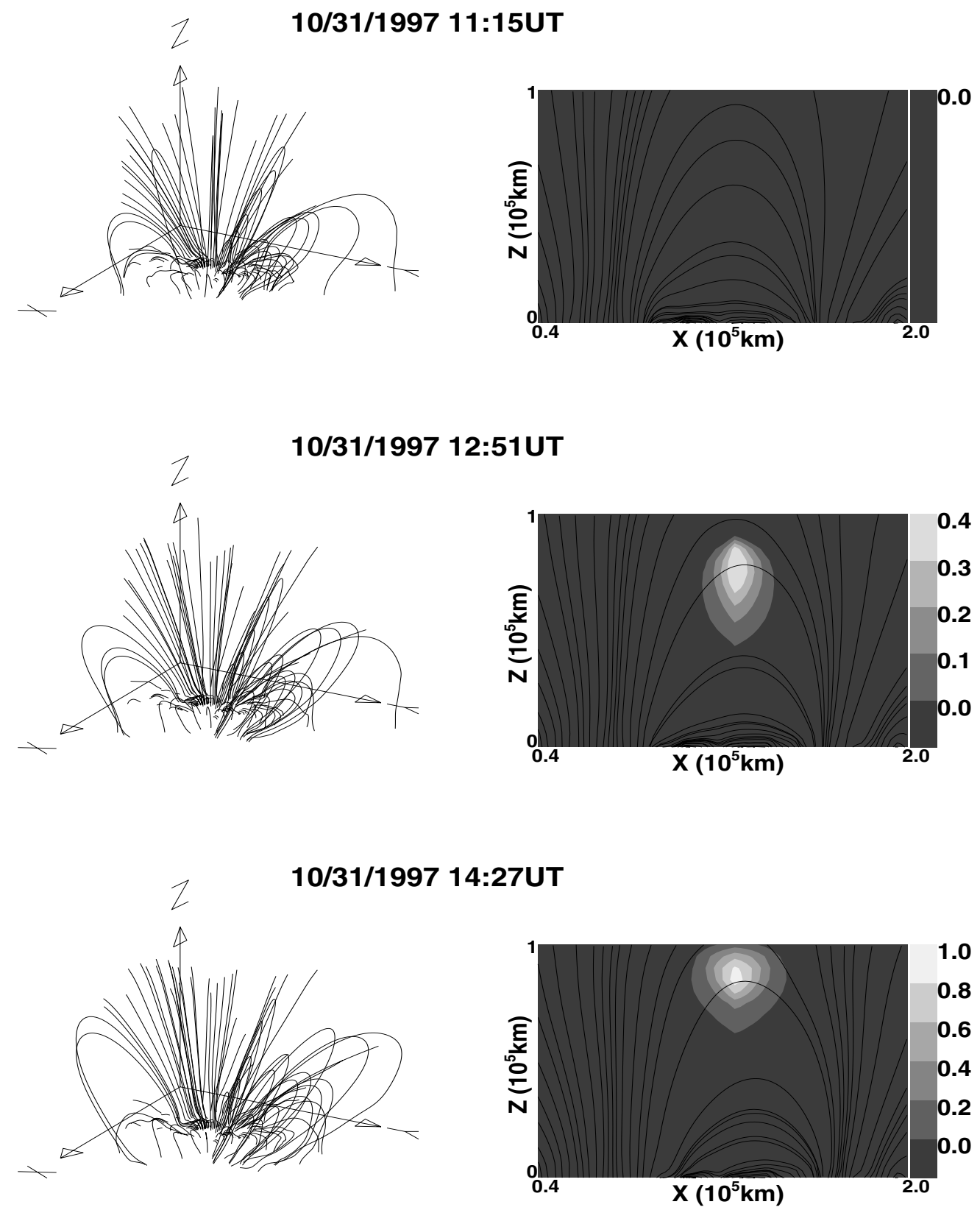

Figure 3. The computed three-dimensional magnetic field evolution (left column) and corresponding field line projection on the $\mathrm{X}-\mathrm{z}$ plane with density enhancement contours $\frac{\left(\rho-\rho_{o}\right)}{\rho_{o}}$ (right column).

suggested by Antiochos, et al. (1999). As such, a coronal mass ejection could be initiated. These features can be seen in Figure 3.

Figure 3 shows the three-dimensional magnetic field evolution in three specific times (left column) and the corresponding projection of these field lines on the x-z plane together with density contours $\frac{\left(\rho-\rho_{o}\right)}{\rho_{o}}$ in gray scale (right column) to show the movement of the plasma blob. By looking at the field lines and density contours on the $\mathrm{x}-\mathrm{z}$ plane 
together with the $L_{s s}$ shown in Table 1 , it is easily recognized that when $L_{s s}$ increases, the field lines rise up and begin to open. Consequently, the mass is lifted up by the field movement. As soon as the field opens, then, the mass will release to form a CME. It should be noted that the initial density shown at 11:15 UT is null, because the background density has been subtracted (i.e. $\frac{\rho-\rho_{o}}{\rho_{o}}$ ).

\section{Summary and Concluding Remarks}

A three-dimensional, time-dependent magnetohydrodynamic (MHD) model for the Active Region (AR) evolution is presented. This newly developed MHD model includes new physics which has usually been neglected. These new physical terms in the governing equations are caused by the introduction of the Sun's differential rotation and meridional flow. These new terms are the inertial centrifugal force and coriolis force in the momentum equation and the cyclonic turbulence effect in the induction equation (Eq. 2.4). In addition, the effect due to random motion of the granules or super-granules is included in the form of an effective diffusion term. This newly developed model is tested by using a specific data set (i.e. SOHO/MDI magnetogram recorded NOAA/AR8100). Results show that the model reproduces observations well (see Fig. 1 and 2). Then, we have employed this model to simulate the non-potential parameters developed by Falconer et al. (2002). Again, the model performed reasonably well (see Table 1).

In summary, some of the important properties of this model can be described as follows:

(a) the new formulation with inclusion of differential rotation and meridional flow give rise to the magnetic shear. It leads to the complexity of the magnetic field features which give understanding of the growth and decay of an active region.

(b) this model has the potential to be utilized to quantify the critical parameters for the initiation of solar eruptive phenomena (i.e. flare/CME), such that a predictive model for solar eruptive events could be achieved.

\section{Appendix A. Bottom Boundary conditions}

This set of boundary conditions are obtained by method of characteristics (Wu \& Wang 1987, Wang 1992) with the assumption of positive vertical velocity and less than characteristic speeds (i.e. Alfven, slow and fast wave speed). These expressions which describe the physical parameters of pressure, density, the components of velocity, and magnetic field vary with time on the boundary are:

$$
\begin{gathered}
\frac{\partial p}{\partial t}=\frac{V_{s}^{2} B+V_{f}^{2} C}{2 V_{A}^{2}\left(V_{f}^{2}-V_{s}^{2}\right)} \\
\frac{p}{\rho^{\gamma}}=\text { const. } \\
\frac{\partial u_{x}}{\partial t}=\frac{B_{y}\left(V_{s}\left(V_{A}^{2}-V_{s}^{2}\right) B-V_{f}\left(V_{f}^{2}-V_{A}^{2}\right) C\right)}{2 B_{z}\left(B_{x}^{2}+B_{y}^{2}\right) V_{s} V_{f}\left(V_{f}^{2}-V_{s}^{2}\right)}+\frac{B_{x} A}{2 B_{z}\left(B_{x}^{2}+B_{y}^{2}\right)} \\
\frac{\partial u_{y}}{\partial t}=\frac{B_{x}\left(V_{s}\left(V_{A}^{2}-V_{s}^{2}\right) B-V_{f}\left(V_{f}^{2}-V_{A}^{2}\right) C\right)}{2 B_{z}\left(B_{x}^{2}+B_{y}^{2}\right) V_{s} V_{f}\left(V_{f}^{2}-V_{s}^{2}\right)}-\frac{B_{y} A}{2 B_{z}\left(B_{x}^{2}+B_{y}^{2}\right)}
\end{gathered}
$$




$$
\begin{gathered}
\frac{\partial u_{z}}{\partial t}=\frac{\left(-V_{s} B+V_{f} C\right)}{2 \rho V_{s} V_{f}\left(V_{f}^{2}-V_{s}^{2}\right)} \\
B_{z}=B_{z o}(x, y, t)(1+a \Delta t)+\sum_{x, y, t} \delta B_{z}(x, y, t) \\
\frac{\partial B_{x}}{\partial t}=\frac{B_{x}\left(\left(V_{A}^{2}-V_{s}^{2}\right) B-\left(V_{f}^{2}-V_{A}^{2}\right) C\right)}{2 V_{A}^{2}\left(V_{f}^{2}-V_{s}^{2}\right)\left(B_{x}^{2}+B_{y}^{2}\right)}+\frac{B_{y} A}{2 V_{A}\left(B_{x}^{2}+B_{y}^{2}\right)} \\
\frac{\partial B_{y}}{\partial t}=\frac{B_{y}\left(\left(V_{A}^{2}-V_{s}^{2}\right) B-\left(V_{f}^{2}-V_{A}^{2}\right) C\right)}{2 V_{A}^{2}\left(V_{f}^{2}-V_{s}^{2}\right)\left(B_{x}^{2}+B_{y}^{2}\right)}-\frac{B_{x} A}{2 V_{A}\left(B_{x}^{2}+B_{y}^{2}\right)}
\end{gathered}
$$

where the coefficients $\mathrm{A}, \mathrm{B}$, and $\mathrm{C}$ are given below.

$$
\begin{aligned}
& A=-\left(u_{z}-V_{A}\right)\left[B_{y} B_{z} \frac{\partial u_{z}}{\partial z}-B_{x} B_{z} \frac{\partial u_{y}}{\partial z}+B_{y} V_{A} \frac{\partial B_{x}}{\partial z}-B_{x} V_{A} \frac{\partial B_{y}}{\partial z}\right] \\
& +\left(B_{x} B_{y} V_{A}-u_{x} B_{y} B_{z}\right) \frac{\partial u_{x}}{\partial x}-\left(B_{x}^{2} V_{A}-B_{x} B_{z} u_{x}\right) \frac{\partial u_{y}}{\partial x}-\frac{B_{y} B_{z}}{\rho} \frac{\partial p}{\partial x} \\
& -\frac{B_{z}}{\rho}\left(B_{x}^{2}+B_{y}^{2}\right) \frac{\partial B_{y}}{\partial x}+B_{x} V_{A} u_{x} \frac{\partial B_{y}}{\partial x}-u_{x} B_{y} V_{A} \frac{\partial B_{x}}{\partial x}-\frac{B_{y} B_{z}^{2}}{\rho} \frac{\partial B_{z}}{\partial x} \\
& +\left(B_{y}^{2} V_{A}-u_{y} B_{y} B_{z}\right) \frac{\partial u_{x}}{\partial y}-\left(B_{x} B_{y} V_{A}-B_{x} B_{z} u_{y}\right) \frac{\partial u_{y}}{\partial y}+\frac{B_{x} B_{z}}{\rho} \frac{\partial p}{\partial y} \\
& +\frac{B_{z}}{\rho}\left(B_{x}^{2}+B_{y}^{2}\right) \frac{\partial B_{x}}{\partial y}-u_{y} B_{y} V_{A} \frac{\partial B_{x}}{\partial y}+u_{y} B_{x} V_{A} \frac{\partial B_{y}}{\partial y}+\frac{B_{x} B_{z}^{2}}{\rho} \frac{\partial B_{z}}{\partial y}, \\
& B=-\left(u_{z}-V_{f}\right)\left[\left(B_{x} B_{z} V_{f} \frac{\partial u_{x}}{\partial z}+B_{y} B_{z} V_{f} \frac{\partial u_{y}}{\partial z}+\rho V_{f}\left(V_{f}^{2}-V_{A}^{2}\right) \frac{\partial u_{z}}{\partial z}\right.\right. \\
& \left.+\left(V_{f}^{2}-V_{A}^{2}\right) \frac{\partial p}{\partial z}+B_{x} V_{f}^{2} \frac{\partial B_{x}}{\partial z}+B_{y} V_{f}^{2} \frac{\partial B_{y}}{\partial z}\right] \\
& -\left(B_{x} B_{z} V_{f} u_{x}+a^{2} \rho\left(V_{f}^{2}-V_{A}^{2}\right)+B_{y}^{2} V_{f}^{2}\right) \frac{\partial u_{x}}{\partial x}-B_{y} V_{f}\left(u_{x} B_{z}+B_{x} V_{f}\right) \frac{\partial u_{y}}{\partial x} \\
& +\rho u_{x} V_{f}\left(V_{f}^{2}-V_{A}^{2}\right) \frac{\partial u_{z}}{\partial x}-\left(\frac{B_{x} B_{z} V_{f}}{\rho}+u_{x}\left(V_{f}^{2}-V_{A}^{2}\right)\right) \frac{\partial p}{\partial x}-u_{x} B_{x} V_{f}^{2} \frac{\partial B_{x}}{\partial x} \\
& -u_{x} B_{y} V_{f}^{2} \frac{\partial B_{y}}{\partial x}-B_{x} V_{f}^{3} \frac{\partial B_{z}}{\partial x}+\left(B_{x} B_{y} V_{f}^{2}-u_{y} B_{x} B_{z} V_{f}\right) \frac{\partial u_{x}}{\partial y} \\
& -\left(u_{y} B_{y} B_{z} V_{f}+a^{2} \rho\left(V_{f}^{2}-V_{A}^{2}\right)+B_{x}^{2} V_{f}^{2}\right) \frac{\partial u_{y}}{\partial y}+\rho V_{f}\left(V_{f}^{2}-V_{A}^{2}\right) u_{y} \frac{\partial u_{z}}{\partial y} \\
& -\left(\frac{B_{y} B_{z} V_{f}}{\rho}-u_{y}\left(V_{f}^{2}-V_{A}^{2}\right)\right) \frac{\partial p}{\partial y}-u_{y} B_{x} V_{f}^{2} \frac{\partial B_{x}}{\partial y}-u_{y} B_{y} V_{f}^{2} \frac{\partial B_{y}}{\partial y} \\
& -B_{y} V_{f}^{3} \frac{\partial B_{z}}{\partial y}+\rho g V_{f}\left(V_{f}^{2}-V_{A}^{2}\right)
\end{aligned}
$$




$$
\begin{array}{r}
C=-\left(u_{z}-V_{s}\right)\left[\left(B_{x} B_{z} V_{s} \frac{\partial u_{x}}{\partial z}+B_{y} B_{z} V_{s} \frac{\partial u_{y}}{\partial z}+\rho V_{s}\left(V_{s}^{2}-V_{A}^{2}\right) \frac{\partial u_{z}}{\partial z}\right.\right. \\
\left.+\left(V_{s}^{2}-V_{A}^{2}\right) \frac{\partial p}{\partial z}+V_{s}^{2}\left(B_{x} \frac{\partial B_{x}}{\partial z}+B_{y} \frac{\partial B_{y}}{\partial z}\right)\right] \\
+\left[a^{2} \rho\left(V_{s}^{2}-V_{A}^{2}\right)+B_{y}^{2} V_{s}^{2}+B_{x} B_{z} V_{s} u_{x}\right] \frac{\partial u_{x}}{\partial x}+B_{y} V_{s}\left(u_{x} B_{z}+B_{x} V_{s}\right) \frac{\partial u_{y}}{\partial x} \\
-\rho u_{x} V_{s}\left(V_{s}^{2}-V_{A}^{2}\right) \frac{\partial u_{z}}{\partial x}+\left[u_{x}\left(V_{s}^{2}-V_{A}^{2}\right)+\frac{B_{x} B_{z} V_{s}}{\rho}\right] \frac{\partial p}{\partial x}+u_{x} B_{x} V_{s}^{2} \frac{\partial B_{x}}{\partial x} \\
+u_{x} B_{y} V_{s}^{2} \frac{\partial B_{y}}{\partial x}+B_{x} V_{s}^{3} \frac{\partial B_{z}}{\partial x}-\left(B_{x} B_{y} V_{s}^{2}-u_{y} B_{x} B_{z} V_{s}\right) \frac{\partial u_{x}}{\partial y} \\
+\left[a^{2} \rho\left(V_{s}^{2}-V_{A}^{2}\right)+B_{x}^{2} V_{s}^{2}+u_{y} B_{y} B_{z} V_{s}\right] \frac{\partial u_{y}}{\partial y}-\rho V_{s}\left(V_{s}^{2}-V_{A}^{2}\right) u_{y} \frac{\partial u_{z}}{\partial y} \\
+\left[\frac{B_{y} B_{z} V_{s}}{\rho}+u_{y}\left(V_{s}^{2}-V_{A}^{2}\right)\right] \frac{\partial p}{\partial y}-u_{y} B_{x} V_{s}^{2} \frac{\partial B_{x}}{\partial y}+u_{y} B_{y} V_{s}^{2} \frac{\partial B_{y}}{\partial y} \\
+B_{y} V_{s}^{3} \frac{\partial B_{z}}{\partial y}+\rho g V_{s}\left(V_{s}^{2}-V_{A}^{2}\right)
\end{array}
$$

Alfvén Speed

Fast MHD Wave Speed

$$
V_{A}=\frac{B_{z}}{\sqrt{4 \pi \rho}},
$$

$$
V_{f}^{2}=\frac{1}{2}\left(\left(a^{2}+b^{2}\right)+\left(\left(a^{2}+b^{2}\right)^{2}-4 a^{2} V_{A}^{2}\right)^{1 / 2}\right),
$$

Slow MHD Wave Speed

$$
V_{s}^{2}=\frac{1}{2}\left(\left(a^{2}+b^{2}\right)-\left(\left(a^{2}+b^{2}\right)^{2}-4 a^{2} V_{A}^{2}\right)^{1 / 2}\right),
$$

with

Sound Speed

$$
b=\sqrt{\frac{B_{x}^{2}+B_{y}^{2}+B_{z}^{2}}{4 \pi \rho}}
$$

$$
a=\sqrt{\gamma R T}
$$

\section{Acknowledgements}

The work performed by STW and AHW is supported by NASA grant NAG5-12843, NSF grant ATM0316115, and AAMU subcontract under NNG04GD59G. DAF is supported by NASA Cooperative Agreement NCC8-200 and NSF grants ATM-0352834 and ATM-0203098. The authors wish to thank Dr. C. D. Fry for reading the manuscript and making useful comments.

\section{References}

Antiochos, S.K., DeVore, C.R., \& Klumchuk, J.A. 1999, ApJ 510, 258

Devore, C.R., Sheeley, N.R., Jr., Boris, J.P., Young, R.T., Jr., \& Harvey, K.L. 1984, Solar Phys. 92,1 
Devore, C. R., Sheeley, N.R., Jr., Boris, J.P., Young, R.T., Jr., \& Harvey, K.L. 1985, Solar Phys 102,41

Devore, C. R., \& Sheeley, N. R., 1987, Solar Phys 108, 47

Falconer, D.A., Moore, R.L. \& Gary, G.A., 2002, ApJ 569, 1016.

Leighton, R.B. 1964, ApJ 140, 1547

McKay, D.H. 2003, Solar Phys 213, 173

Hathaway, D.H 1996 ApJ 460, 1027

McIntosh, P.S. \& Wilson, P.R. 1985 Solar Phys 99, 59

Parker, E.N. 1979 in: Cosmic Magnetic Fields (Oxford University Press, England), p. 509

Schrijver, C.J. 2001 ApJ 547, 475

Schrijver, C.J. \& Title, A.M. 2002 ApJ 551, 1099

Sheeley, N.R., Jr., Devore, C.R., \& Boris, J. P. 1985 Solar Phys 98, 219

Sheeley, N.R., Jr., \& Devore, C. R. 1986 Solar Phys 103, 203

Wang, A.H. 1992, PhD thesis, University of Alabama in Huntsville.

Wang, H.M. 1988 Solar Phys 116, 1

Wang, H.M., Spirock, T.J., Qiu, J., Ji, H., Yurchyshyn, V., Moon, Y-J., Denker, C. \& Goode, P.R. 2002 ApJ 576, 497

Wang, H.M., Qiu, J., Jing, J. Spirock T. J., Yurchyshyn, V., Abramenko, V., Ji, H. \& Goode, P.R. 2004 ApJ 605, 931

Wang, Y.-M. \& Sheeley, N. R., Jr., 1991 ApJ 375, 761

Wilson, P.R. \& McIntosh, P.S. 1991 Solar Phys 136, 221

Wu, S.T., Wang, J.F. 1987 Comp. Methods in Appl. Mech. 64, 267

Wu, S.T., Yin, C.L., McIntosh, P.S. \& Hildner, E. 1993 in: H.Zirin, G. Ai \& H.M. Wang (eds.), The Magnetic and Velocity Fields of Solar Active Regions, ASP Conference Series (New York: AIP), vol. 46, p. 98

\section{Discussion}

SCHMIEDER: I appreciate your model. The total flux follows the observed total flux. The shear parameter $\alpha$ however is increasing during all of your time sequences

Wu: In the example of Falconer, the CME arrives 5 days later. The simulation does not reach the phase of eruption.

Kuтснму: Usually, when we observe an increase of the total magnetic flux we think about a flux emergence process of fluxes produced in sub-photospheric layers. In your case, you produce an increase of the flux using surface phenomena. How can one decide which phenomenon is more important and do they have the same sign of variation?

WU: In the model, we are able to accommodate sub-photospheric effect through the timedependent characteristic boundary included in the Appendix. In the present calculation, we prescribe the flux emergence according to a receipt given in Eq. (A6) on the basis of the MDI observation.

GRECHNEV: If your first plot of the total magnetic flux versus time, the difference of the observed and calculated values is a quasi-periodic function, which can be of observational origin. If this quasi-periodic difference can be eliminated, the coincidence of the calculated and observed values would still be there.

Wu: Yes, you are correct. 